\title{
The Study on thermodynamics of martensitic transformation in Laser additive manufacturing alloy steel
}

\author{
Lianyun $\mathrm{Xi}^{1, \mathrm{a}}$, Suiyuan Chen ${ }^{1, \mathrm{~b}, ~}{ }^{*}$, Jing Liang ${ }^{1, \mathrm{c}}$, Tong Cui ${ }^{1, \mathrm{~d}}$, Changsheng Liu ${ }^{1, \mathrm{e}}$ \\ Key Laboratory for Anisotropy and Texture of Materials(Ministry of Education), School of Materials and \\ Engineering, Northeastern University, Shenyang 110819, China \\ a18341272565@163.com, ,, ${ }^{\text {c }}$ chensy@atm.neu.edu.cn, ${ }^{\mathrm{C} L i a n g}$ j@atm.neu.edu.cn, \\ dcuit@smm.neu.edu.cn, ${ }^{\mathrm{e}}$ csliu @mail.neu.edu.cn
}

Keywords: laser additive manufacturing; empirical formula; 24CrNiMo alloy steel; thermodynamic of martensitic transformation.

\begin{abstract}
Based on the characteristics of metallurgical non-equilibrium solidification behavior during laser additive manufacturing alloy steel, the basic theory of thermodynamics of martensitic transformation precipitating in the process of selective laser melting deposition of alloy steel were introduced, then binary, ternary and multicomponent alloy steel systems thermodynamic evolution process were revealed. Combined with selective laser melting deposited 24CrNiMo alloy steel experiment, laser additive manufacturing 24CrNiMo alloy steel CCT curve and the thermodynamic data-martensite transformation starting temperature were simulated. Simulated by using simulation software and calculated with empirical formula the temperature of martensitic transformation starting point are $366.6^{\circ} \mathrm{C}$ and $361.102^{\circ} \mathrm{C}$ respectively. It is expected that these studies can provide theoretical reference for laser additive manufacturing brake disc of High-speed rail.
\end{abstract}

\section{Introduction}

As one of the revolutionary frontier technologies in the manufacturing field laser additive manufacturing has attracted a great deal of attention and has been developing rapidly worldwide. High speed rail is the strategic leading industry of people's livelihood and its brake disc is key parts of the core equipment guaranteeing the safe and stability of the train operation. Being the traditional method of producing brake discs, casting has some problems such as complex process, long production cycle, short product life. Instead, laser additive manufacturing have distinctive features of one forming for complex structure and short process flow. However, it is a non-equilibrium solidification process with complex organization phase transition behavior. Meanwhile due to cumulative effect of heat from multilayer cladding studying thermodynamics has an important effect on the structure and performance of the final forming alloy.

So far, there is little research on non-equilibrium solidification behavior of alloy steel produced by laser additive manufacturing. As one of the most important phases in alloy steel, martensitic transformation mechanism is the basis for successful manufacture alloy steel with good microstructure and properties. However, process design closely related to the non balance technology is still mainly rely on empirical or semi quantitative theory not to meet the current requirements of alloy steel by laser material manufacturing [1]. It is urgent for people to make new 
breakthroughs in solidification theory research.

Thermodynamic development of martensitic precipitating from 24 CrNiMo alloy steel, one material is used to manufacture high speed rail brake disc. This paper analyzed basic theory relative to the mechanism of martensitic phase, simulated the temperature of martensitic transformation starting point (Ms) and CCT curves, and calculated Ms temperature with empirical formula, expected to provide a valuable basic theory for high speed rail brake disc made of laser additive manufacturing.

\section{The development of thermodynamics martensitic transformation}

Due to rapid heat and cool (cooling rate is above $10^{3 \circ} \mathrm{C} / \mathrm{s}$ ) non-equilibrium phase martensite can be observed in laser selective deposited 24CrNiMo alloy steel. Thus, thermodynamic of martensitic transformation are introduced, including thermodynamic model, such as Neural network model, Super member model [3] etc. Hsu T. Y. [2] defined the physical meaning of Gibbs free energy of martensitic transformation, studied martensitic transformation thermodynamics systematically, including binary, ternary alloy steel system. In recent years, some researchers have studied Ms temperature of polynary system iron alloy. Using software to simulate the thermodynamics of martensitic transformation has some reference significance for the experimental study of high-speed brake disc.

\subsection{Thermodynamics of martensitic formation in binary alloy steel}

While studying the binary alloy steel, Hsu T Y [2] gave the Gibbs free energy formula of martensitic transformation as follows:

$$
\Delta G^{\gamma \rightarrow m}=\Delta G^{\gamma \rightarrow \alpha}+\Delta G^{\alpha \rightarrow m}
$$

In Eq.(1), $\Delta G^{\gamma \rightarrow \alpha}$ is the energy to extend the nuclear structure of the somatic structure, and $\Delta G^{\alpha \rightarrow m}$ is the energy to made the nuclear structure martensite. Hsu T Y defined the temperature at which the Gibbs free energy of martensitic transformation is zero as the starting point of martensitic transformation. when the value of first part in Eqs.(1) is zero the corresponding temperature is $T_{0}$. D-value between the Gibbs free energy between $T_{0}$ and $M_{s}$ temperature is called the martensitic transformation driving force, that is $-\Delta G^{\gamma \rightarrow \alpha}=0$ or $\Delta G^{\alpha \rightarrow m}=0$.

The phase change driving force $\left(\Delta G^{\gamma \rightarrow \alpha}\right)$ can be calculated by

$$
\Delta G^{\gamma \rightarrow \alpha}=(1-X c) \Delta G_{F e}^{\gamma \rightarrow \alpha}+(1-X c) R T \ln \frac{\alpha_{F e}^{a}}{\alpha_{F e}^{\gamma}}+X c R T \ln \frac{\gamma_{c}^{a}}{\gamma_{c}^{\gamma}}
$$

In Eq.(2), $\alpha_{i}^{j}$ is the activity of i element in the Solid solution $\mathrm{j}$, correspondingly $\gamma$ is the activity coefficient, $\mathrm{X}_{\mathrm{c}}$ is mass percentage of element $\mathrm{C}$.

Using geometric model to calculate the activity value of iron element in ferrite and austenite is feasible. Pure iron Gibbs free energy change can not only be calculated through a variety of models, but also can be calculated by the latest thermodynamic data. In order to calculate $\Delta G^{\alpha \rightarrow m}$ Hsu T Y derived the following formulas:

$$
\Delta G^{\alpha \rightarrow m}=\varphi V_{m} \sigma_{M_{S}}+\sum \Gamma
$$

Combined the third and fourth equations as well as the above data, the following formula can be obtained:

$$
\Delta G^{\gamma \rightarrow m}=\Delta G^{\gamma \rightarrow \alpha}+5\left[13+280 X_{C}+0.02\left(800-M_{S}\right)\right]+217
$$

\subsection{Thermodynamics of martensitic transformation in ternary alloy steel}

Considering the Fisher model and the binary system regular solution model, a ternary regular solution model was proposed [2]:

$$
\Delta G_{F e-x-c}^{\gamma \rightarrow \alpha}=\left(1-X_{c}-X_{i}\right) \Delta G_{F e}^{\gamma \rightarrow \alpha}+X_{c} R T \ln \frac{\gamma_{c}^{a}}{\gamma_{c}^{\gamma}}+X_{i} \Delta G_{i}^{\gamma \rightarrow \alpha}+\Delta \Omega^{\gamma \rightarrow \alpha}
$$

Considering Fisher--Hsu method and Zener two parameteric method $\Delta G_{F e-x-c}^{r \rightarrow \alpha}$ was derived as: 


$$
\begin{aligned}
& \Delta G_{F e-\chi-c}^{\gamma \rightarrow \alpha}=\left(1-X_{C}\right)\left[\Delta G_{F e}^{\gamma \rightarrow \alpha}\left(T-100 X_{i} \Delta T_{\text {mag }}\right)+141 X_{i}\left(\Delta T_{\text {mag }}-\Delta T_{N, M}\right)\right] \Delta G_{F e}^{\gamma \rightarrow \alpha} \\
& +\left(1-X_{c}\right) \frac{1}{5} R T\left[3 \ln \frac{3-8 X_{c}}{3\left(1-X_{c}\right)}-\ln \frac{1-6 X_{C}}{1-X_{C}}\right]+X_{C} R T \ln \frac{\gamma_{c}^{\alpha}}{\gamma_{c}^{\gamma}}+\Delta G^{*}
\end{aligned}
$$

Where $\Delta G^{*}=-2.13+10 \wedge 5 x_{c}^{2}+9.13 x_{c} T \quad \mathrm{~J} / \mathrm{mol}$

It is feasible to estimate the value of $\Delta G^{\alpha \rightarrow m}$ and then calculate the Ms and martensitic transformation driving force using the same method as binary iron alloys.

\subsection{Thermodynamics of martensitic transformation in multicomponent alloys}

It is expected to generalized formula of the ternary alloy by using super member model [3] to calculate thermodynamics of martensitic transformation in multicomponent alloys. Fe- $\Sigma X_{i}$ is regarded as a super member $\mathrm{S}$ in the alloy of $\mathrm{Fe}-\Sigma \mathrm{X}_{\mathrm{i}}-\mathrm{C}\left(\mathrm{X}_{\mathrm{i}}=\mathrm{Si}, \mathrm{Mn}, \mathrm{Cr}, \mathrm{Ni}, \mathrm{Mo}\right.$, Ti ect.). And then it is similar to Fe-C alloy. So multicomponent alloys thermodynamics can be calculated by using the ternary alloy thermodynamics model .

The coresponding $\mathrm{a}_{\mathrm{Fe}}{ }^{\gamma}, \mathrm{a}_{\mathrm{Fe}}^{\mathrm{a}}$ and $\Delta G_{F \mathrm{~F}}{ }^{\gamma \rightarrow \alpha}$ in the binary alloy model can be substituted by $\mathrm{a}_{\mathrm{s}}{ }^{\gamma}, \mathrm{a}_{\mathrm{s}}{ }^{\mathrm{a}}$ and $\Delta G S^{\gamma \rightarrow \alpha}$ respectively. Their calculations are as follows:

$$
\begin{gathered}
\ln \alpha_{s}^{a}=\frac{3}{z_{\alpha}-3} \ln \frac{3-z_{\alpha} x_{\alpha}}{3\left(1-x_{\alpha}\right)} \\
\ln \alpha_{s}^{a}=\frac{3}{z_{\alpha}-3} \ln \frac{3-z_{\alpha} x_{\alpha}}{3\left(1-x_{\alpha}\right)} \\
\Delta G_{S}^{\gamma \rightarrow \alpha}=141 \sum x_{i}\left(\Delta T_{M}^{i}-\Delta T_{N M}^{i}\right)+\Delta G_{F e}^{\gamma \rightarrow \alpha}\left\{T-100 \sum x_{i} \Delta T_{M}^{i}\right\}
\end{gathered}
$$

\subsection{Empirical formula of calculating Ms temperature in multicomponent alloy}

Using thermodynamic model to calculate directly Ms temperature of SLM deposited 24CrNiMo alloy steel is more complex and the task is heavy due to more alloying elements. To simply calculation and reduce work difficulty Ms temperature can be calculated with empirical formula. More importantly, the value can be reference for selective laser melting deposited 24CrNiMo alloy steel experiment. Then continue studying its thermodynamics model to lay the theoretical foundation for the experiment of the high speed rail brake disc by Laser additive manufacturing technology. Some Empirical formulas [4] of calculating Ms temperature are as follows:

Table1 Empirical formula of calculating Ms temperature

\begin{tabular}{lll}
\hline Empirical formula & scope of application \\
\hline $\mathrm{M}_{\mathrm{s}}=539-423 \mathrm{C}-30.4 \mathrm{Mn}-12.1 \mathrm{Cr}-17.7 \mathrm{Ni}-7.5 \mathrm{Si}-7.5 \mathrm{Mo}+10 \mathrm{Co}$ & & High-alloy steel \\
$\mathrm{M}_{\mathrm{s}}=499-292 \mathrm{C}-32.4 \mathrm{Mn}-22 \mathrm{Cr}-16.2 \mathrm{Ni}-10.8 \mathrm{Si}-10.8 \mathrm{Mo}+10 \mathrm{Co}$ & $(10)$ & low-alloy steel \\
$\mathrm{M}_{\mathrm{s}}=767.7-305.4 \mathrm{C}-30.6 \mathrm{Mn}-8.9 \mathrm{Cr}-16.6 \mathrm{Ni}-14.5 \mathrm{Si}+2.4 \mathrm{Mo}+53 \mathrm{~V}$ & $(11)$ & Complex component \\
$+8.58 \mathrm{Co}+40.4 \mathrm{Al}+7.4 \mathrm{~W}-11.3 \mathrm{Cu}+510.4 \mathrm{Nb}$ & & alloy steel \\
\hline
\end{tabular}

Using equation 12 to calculate Ms temperature of low-alloy steel is proved to be closer to the experimental value, and its error is less than $11.5^{\circ} \mathrm{C}$ [5]. According to neural network [4] model it achieved empirical formula 10 , which is suitable for multicomponent alloy steels, but is limited strictly by the content of alloying elements. Albin Stormvinter [6] etc. introduced the relationship between Ms temperature and martensitic transformation driving force at the Ms temperature:

$$
\Delta G_{m}^{* \gamma \rightarrow \alpha}=3857.7-3.3414 M_{s}(K)
$$

Ms temperature not only can be calculated by using simple regression empirical formula, but also 
can be calculated by using the simulation software (Jmatpro). They provide some reference for the experiment of the high speed rail brake disc by laser additive manufacturing technology.

\section{Simulation and calculation of martensitic phase in $24 \mathrm{CrNiMo}$ alloy steel by laser selective melting}

The experimental parameters are as follows. Laser power is $2200 \mathrm{~W}$, scanning rate is $8 \mathrm{~mm} / \mathrm{s}$, defocusing distance is 304. Fig.1(a) and (b) are the metallographic and SEM photographs of the 24CrNiMo alloy sample respectively. It is seen that there exist Martensitic phase in the 24CrNiMo alloy during non-equilibrium cooling process from these two pictures.

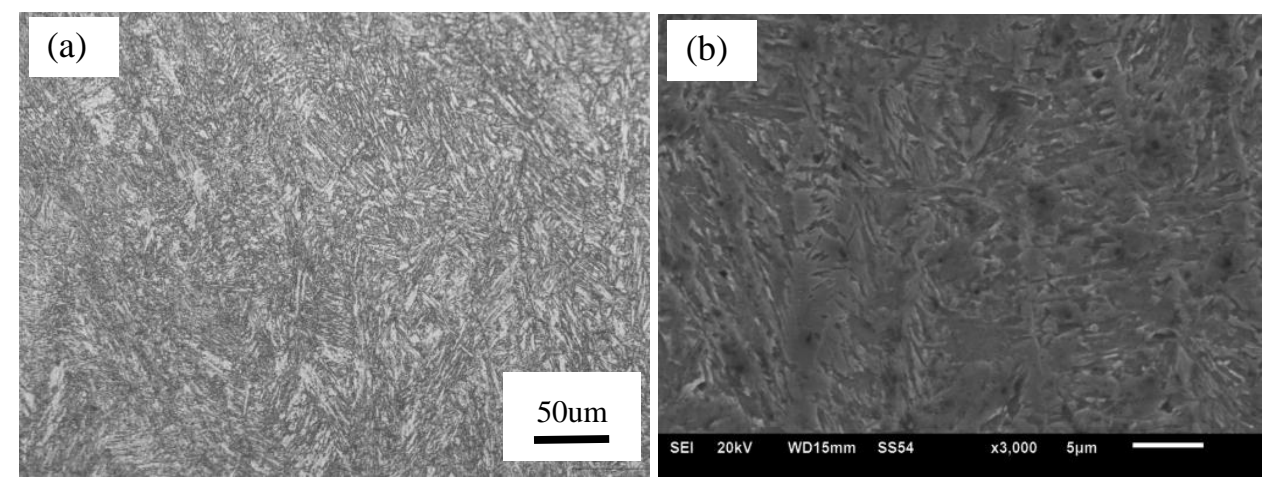

Fig. 1 metallographic and SEM photographs of the

24CrNiMo alloy by laser selective melting

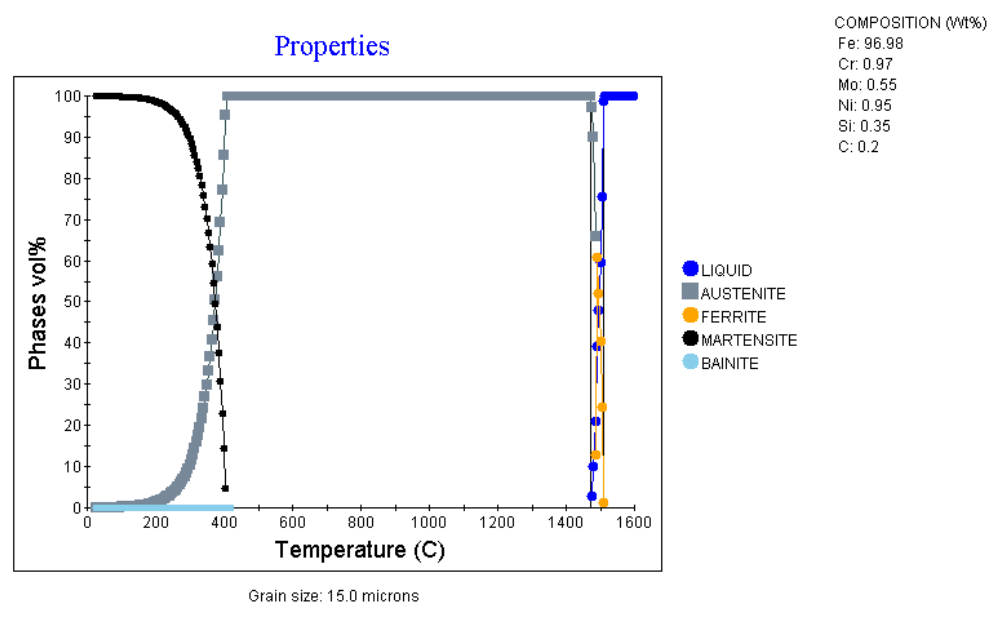

Fig. 2 Relation schema between phase content and temperature of SLM deposited 24CrNiMo alloy steel

Fig. 2 is a non-equilibrium phase diagram of 24CrNiMo alloy steel simulated by Jmatpro software, with a cooling rate of $1000^{\circ} \mathrm{C} / \mathrm{S}$ based on experiment. Fig. 3 is a CCT curve of $24 \mathrm{CrNiMo}$ alloy steel simulated by the software. It can be seen from fig. 2 and fig. 3 that the martensitic phase of the alloy steel is precipitated in the rapid cooling condition. The Ms temperature of 24CrNiMo alloy can be simulated using the relationship between martensitic transformation and the hardness module in the Jmatpro software. It is $366.6^{\circ} \mathrm{C}$. The temperature calculated with the empirical formula

(Eq.10) is $361.102^{\circ} \mathrm{C}$. 


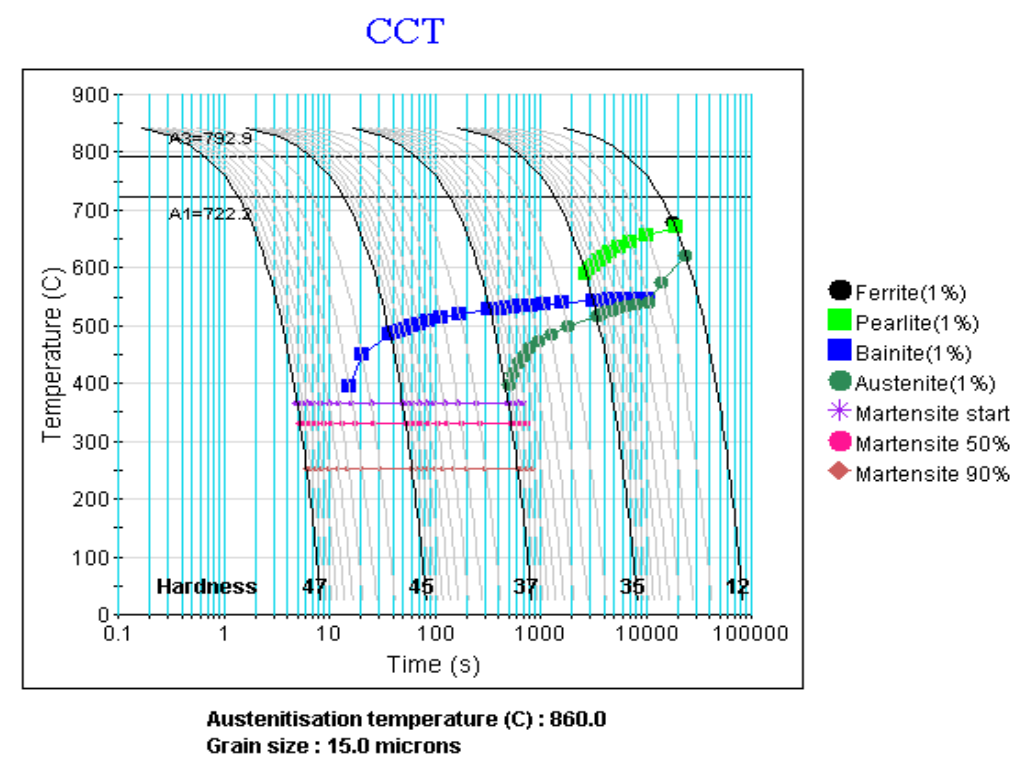

Fig.3 CCT curve of laser selective melting deposited 24CrNiMo alloy steel

\section{Conclusions}

A simulation of the selective laser melting of 24CrNiMo alloy steel martensite start temperature is $366.6^{\circ} \mathrm{C}$, and calculated temperature by using empirical formula is $361.102^{\circ} \mathrm{C}$. This value has positive reference value for the high speed rail brake disc experiment. Describing of the thermodynamic has certain guiding significance for selective laser melting of 24CrNiMo alloy steel thermodynamics of martensitic transformation, hoping to generalize the martensitic transformation thermodynamics model in the ternary alloy by super member algorithm. Ensuring it can be applied to the martensitic transformation of 24CrNiMo alloy steel, and then lay the foundation for the manufacture of high speed rail brake disc with laser additive manufacturing.

\section{Acknowledgements}

This work was financially supported by National Key R\&D Program of China (2016YFB1100201), National Natural Science Foundation of Liaoning United fund (U1508213).

\section{References}

[1] H.F. Wang, K.Wang, W.W. Kuang , Chinese Science: Technical Science, 45(2015) 358-376.

[2] T. Y. Hsu, Martensitic transformation and martensite, Second ed., Science Press, Bei jing,1999.

Reference to a chapter in an edited book: chapter 6 .

[3] Y.Wang , K. Zhao ,G.Tang, Heat Treatment of Metals, 38(2013)1-5.

[4] P. Liu, L. He, Y.X. Zhange, Development and Application of Materials, 30(2015) 88-92.

[5] C. Liu, Z. Zhao, D.O. Northwood, Journal of Materials Processing Tech, 113(2001) 556-562.

[6] A. Stormvinter, A. Borgenstam, J. Ågren, Metallurgical and Materials Transactions, 43( 2012) 3870-3879. 